Z Rheumatol 2014 · 73:113-114

DOI 10.1007/s00393-013-1262-2

Online publiziert: 21. März 2014

๑) Springer-Verlag Berlin Heidelberg 2014
E. Edelmann ${ }^{1} \cdot$ W. Mau ${ }^{2}$

${ }^{1}$ Rheumatologische Gemeinschaftspraxis, Bad Aibling

${ }^{2}$ Institut für Rehabilitationsmedizin, Medizinische Fakultät der

Martin-Luther-Universität Halle-Wittenberg, Halle (Saale)

\title{
Rheumatologische Versorgungsforschung im internationalen Kontext
}

Internationale Daten zeigen in den letzten beiden Jahrzehnten im Zusammenhang mit erheblichen Fortschritten der Therapie deutliche Verbesserungen der Manifestationen und Folgen entzündlich-rheumatischer Erkrankungen wie Krankheitsaktivität, Alltagseinschränkungen, Erwerbstätigkeit und psychische Belastungen [2, 3]. Dies gilt v. a: für Länder mit hohem Bruttosozialprodukt [4]. Daten aus dem Ausland sind deshalb und wegen unterschiedlicher Systeme der sozialen Sicherung und Gesundheitsversorgung nicht ohne Weiteres auf Deutschland übertragbar. Das deutsche Gesundheitssystem ist bisher v. a. geprägt durch die Sektoren der

- von Vertragsärzten in Praxen, von Klinikern in ermächtigten Ambulanzen, in Instituts-, in \$-116b- und Universitätsambulanzen erbrachten ambulanten Leistungen;

- in Krankenhäusern insbesondere akutstationär durchgeführten Diagnostik und Therapie sowie

- in Rehabilitationseinrichtungen stattfindenden Versorgung.

Deshalb hat die Deutsche Gesellschaft für Rheumatologie (DGRh) in ihrem Memorandum zur rheumatologischen Versorgung von akut und chronisch Rheumakranken in Deutschland im Jahr 2008 Strukturen, Defizite und Handlungsempfehlungen mit Bezug auf diese Versorgungssektoren dargestellt [1]. Inzwischen sind neue versorgungsrelevante Daten verfügbar. Ferner zeichnen sich Ansätze zur Überwindung von Sektorengrenzen ab. Diese aktuellen Daten aus der rheumatologischen Versorgungsforschung werden im vorliegenden Leitthemenheft präsentiert und - soweit möglich - internationalen Entwicklungen gegenübergestellt.

Der erste Beitrag (A. Zink) fasst exemplarisch für die rheumatoide Arthritis die neuesten epidemiologischen Daten aus der Kerndokumentation und Medikamentenregistern zusammen. Neben der Prävalenz und Inzidenz werden Verbesserungen der rheumatologischen Mitbetreuung mit höherem Erreichungsgrad, früherer Erstvorstellung und intensiverer medikamentöser Therapie dargestellt. Damit einher gehen Reduktionen der Krankheitsaktivität und schwerer erosiver Verläufe sowie erhöhte Raten für die Remission, gute Funktionsfähigkeit und höhere Erwerbsbeteiligung.

Der zweite Beitrag (E. Edelmann) befasst sich mit der Entwicklung der ambulanten Versorgung. Trotz der zunehmenden Zahl an ambulant tätigen Rheumatologen, Förderung der Früharthritis-Sprechstunde durch Selektivverträge in einzelnen Regionen und durch einzelne Krankenkassen sind lange Wartezeiten in den Praxen und Ambulanzen die Regel. Im Vergleich mit 8 weltweiten Industrienationen nimmt Deutschland hinsichtlich der Zahl der Rheumatologen pro 100.000 Einwohner einen mittleren Platz ein. Diagnosestatistiken der Kassenärztlichen Bundesvereinigung zeigen in den letzten Jahren eine deutliche Zunahme der von den fachärztlichen internistischen Rheumatologen betreuten Patien- ten mit chronisch-entzündlichen Rheumaformen. Eine sektorenübergreifende, budgetfreie Versorgung nach $\$ 116 \mathrm{~b}$, die sogenannte ambulante spezialfachärztliche Versorgung (ASV), an der gleichermaßen niedergelassene wie in der Klinik tätige Rheumatologen teilhaben können, steht für Ende des Jahres 2014 in Aussicht. Qualitätsorientierte Selektivverträge mit dem Ziel einer integrierten, umfassenden und sektorenübergreifenden Versorgung von Patienten mit chronisch-entzündlichen Rheumaformen werden bundesweit verhandelt. Eine größere Nachhaltigkeit in der Versorgung, eine bessere Vergütung rheumatologischer Leistungen, eine intensivere und umfänglichere rheumatologische Versorgung von mehr Patienten ist durch die ASV und durch Selektivverträge zu erwarten.

Der dritte Beitrag (J. Kekow) beschäftigt sich mit der akutstationären rheumatologischen Versorgung. Auch im stationären Bereich besteht bundesweit ein Mangel an qualifizierten rheumatologischen Einrichtungen mit der Folge einer nicht zielführenden Behandlung eines Großteils der Patienten in Kliniken ohne rheumatologische Kompetenz. Das Vergütungssystem im stationären Bereich (DRG-System) erscheint mit Ausnahme einer Fallpauschale zur rheumatologischen Komplextherapie wenig geeignet, die Versorgung von Patienten mit chronisch-entzündlichen Rheumaformen abzubilden. Mit der sektorenübergreifenden Versorgung im Rahmen der ASV wird auch für den stationären Bereich eine Chance gesehen, die rheumatologi- 
sche Versorgung umfassend zu verbessern. Es wird eine besondere Verpflichtung der stationären Rheumatologie gesehen, für eine ausreichende Weiterbildung zu sorgen. Die Zahl der Anerkennungen für das Fachgebiet Rheumatologie stagniert seit Jahren bzw. ist zum Teil rückläufig. Hilfreich ist ein laufendes Projekt von DGRh, BDRh und VRA, mit dem Weiterbildungsplätze finanziell gefördert werden. Mehr Universitätsabteilungen mit eigenem Budget sind erforderlich, um genügend Weiterbildungsstellen zu ermöglichen.

Die Mitglieder der Kommission Rehabilitation und Sozialmedizin der DGRh (W. Mau et al.) stellen im vierten Artikel aus den letzten ein bis zwei Dekaden rückläufige Trends von Leistungen zur medizinischen Rehabilitation der Deutschen Rentenversicherung zusammen. Erhebliche regionale Unterschiede stationärer Rehabilitation werden aufgedeckt. Daten von einer der größten Krankenkassen zeigen deutlich seltenere Rehabilitationsleistungen im Vergleich zur Deutschen Rentenversicherung. Struktur- und Prozessqualität der Rehabilitation sind in Einrichtungen mit hohen Zahlen von Patienten mit entzündlich-rheumatischen Krankheiten deutlich höher als bei geringen Belegungen mit diesen Erkrankungen. In der ambulanten rheumatologischen Versorgung werden Defizite funktions-/bewegungsorientierter Therapie mit großen Unterschieden zwischen den Praxen identifiziert. Möglichkeiten rehabilitativer Interventionen aus anderen europäischen Ländern werden exemplarisch vorgestellt.

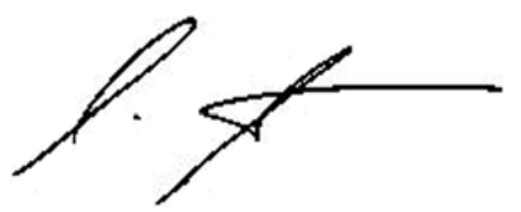

Dr. Edmund Edelmann

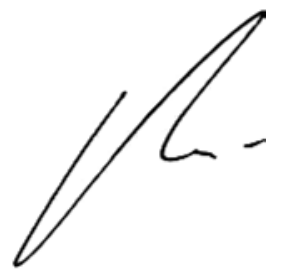

Prof. Dr. Wilfried Mau

\section{Korrespondenzadressen}

\section{Dr. E. Edelmann}

Rheumatologische Gemeinschaftspraxis

Lindenstr. 2, 83043 Bad Aibling

edelmann@bdrh.de

\section{Prof. Dr. W. Mau}

Institut für Rehabilitationsmedizin,

Medizinische Fakultät der

Martin-Luther-Universität Halle-Wittenberg

06097 Halle (Saale)

wilfried.mau@medizin.uni-halle.de

Interessenkonflikt. E. Edelmann und W. Mau geben an, dass kein Interessenkonflikt besteht.

\section{Literatur}

1. Deutsche Gesellschaft für Rheumatologie (2008) Memorandum: Rheumatologische Versorgung von akut und chronisch Rheumakranken in Deutschland. http://dgrh.de/?id=8878. Zugegriffen: 13.02.2014

2. Hallert $E$, Husberg M, Bernfort L (2012) The incidence of permanent work disability in patients with rheumatoid arthritis in Sweden 1990-2010: before and after introduction of biologic agents. Rheumatology 51:338-346

3. Overman $C L$, Jurgens $M S$, Bossema $E R$, Jacobs JWG, Bijlsma JWJ, Geenen R (2013). Patients with rheumatoid arthritis nowadays are less psychologically distressed and physically disabled than patients two decades ago. Arthritis Care Res (Holboken) doi10.1002/acr.22211

4. Sokka T, Kautiainen H, Pincus T, Toloza S, Da Rocha Castelar Pinheiro G, Lazovskis J, et al. (2009) Disparities in rheumatoid arthritis disease activity according to gross domestic product in 25 countries in the QUEST-RA database. Ann Rheum Dis 68:16661672

\section{EULAR-Textbook on Systemic Sclerosis}

London, Zürich: BMJ Publishers, EULAR 2013, (ISBN 978-1-905545-38-4), 70.00 EUR

Es gibt wenig seltene Erkrankungen der Rheumatologie, die soviel Aufmerksamkeit auf sich ziehen konnten und können, dass ein eigenens Lehrbuch für diese unter der Führung der europäischen rheumatologischen Gesellschaft EULAR erstellt werden konnte. Mit dem EULAR-Textbook on Systemic Sclerosis, welches von zwei führenden Rheumatologen, die sich seit langer Zeit mit der systemischen Sklerose beschäftigen, Professor Erika Huchulla und Professor Laszlo Czirjak, koordiniert und herausgegeben wurde, ist ein neues Standardwerk geschaffen worden, welches mit Sicherheit zu den täglichen Nachschlagewerken in der rheumatologischen Praxis gehören dürfte. Die Besonderheit dieses Lehrbuches ist, dass sich nahezu alle weltweit in diesem Gebiet aktiven Wissenschaftler und Kliniker zusammengefunden haben, um nicht nur ein reines Übersichtsbuch, sondern vor allem auch ein reich bebildertes Lehrbuch zu schaffen, um den jungen Kollegen und den sich in der Weiterbildung befindlichen Rheumatologen möglichst anschaulich und trotzdem hoch detailliert die Facetten dieser vielfältigen Erkrankung vor Augen zu führen und gleichzeitig praxisnah zu erklären.

Das Lehrbuch selbst ist in 10 Hauptkapitel unterteilt, die von der Epidemiologie und Pathophysiologie ausgehend die einzelnen betroffenen Organe und Organsysteme mit ihren Besonderheiten bezüglich Diagnose, Differenzialdiagnose und Therapie darstellen und so für jede klinische Situation eine soweit möglich passende Lösung bereit halten. Auch sogenannte cutting-edgeStrategien, die jetzt erst in die klinische Routine eingeführt werden, sind ausführlich beschrieben, so dass wie für seltene Erkrankungen häufig notwendig auch sehr ungewöhnliche Konstellationen in der Praxis mit den entsprechenden Informationen unterfüttert werden. Obwohl das Buch selbst aus nachvollziehbaren Gründen auf englisch geschrieben ist, ist es so gut zu lesen, dass dies kein Hinderungsgrund sein dürfte, ihm nicht umgehend einen Platz in der eigenen Praxisbibliothek zuzuweisen.

Professor Dr. Ulf Müller-Ladner (Bad Nauheim) 